Subscriber access provided by Caltech Library

\title{
Article
}

\section{Machine-Learning Methods Enable Exhaustive Searches for Active Bimetallic Facets and Reveal Active Site Motifs for CO2 Reduction}

Zachary W Ulissi, Michael T Tang, Jianping Xiao, Xinyan Liu, Daniel A. Torelli, Mohammadreza Karamad, Kyle Cummins, Christopher Hahn, Nathan S. Lewis, Thomas F. Jaramillo, Karen Chan, and Jens K. Norskov

ACS Catal., Just Accepted Manuscript • DOI: 10.1021/acscatal.7b01648 • Publication Date (Web): 27 Jul 2017

Downloaded from http://pubs.acs.org on July 27, 2017

\section{Just Accepted}

"Just Accepted" manuscripts have been peer-reviewed and accepted for publication. They are posted online prior to technical editing, formatting for publication and author proofing. The American Chemical Society provides "Just Accepted" as a free service to the research community to expedite the dissemination of scientific material as soon as possible after acceptance. "Just Accepted" manuscripts appear in full in PDF format accompanied by an HTML abstract. "Just Accepted" manuscripts have been fully peer reviewed, but should not be considered the official version of record. They are accessible to all readers and citable by the Digital Object Identifier (DOI®). "Just Accepted" is an optional service offered to authors. Therefore, the "Just Accepted" Web site may not include all articles that will be published in the journal. After a manuscript is technically edited and formatted, it will be removed from the "Just Accepted" Web site and published as an ASAP article. Note that technical editing may introduce minor changes to the manuscript text and/or graphics which could affect content, and all legal disclaimers and ethical guidelines that apply to the journal pertain. ACS cannot be held responsible for errors or consequences arising from the use of information contained in these "Just Accepted" manuscripts. 


\title{
Machine-Learning Methods Enable Exhaustive Searches for Active Bimetallic Facets and Reveal Active Site Motifs for $\mathrm{CO}_{2}$ Reduction
}

Zachary W. Ulissi, ${ }^{\dagger, \dagger}$ Michael T. Tang,,,$+ \dagger$ Jianping Xiao,,,$+ \dagger$ Xinyan Liu,,,,$+ \dagger$ Daniel A. Torelli,,$\uparrow$ Mohammadreza Karamad, ${ }^{\ddagger}$ Kyle Cummins, ${ }^{\mathbb{1}, \dagger}$ Christopher Hahn,,,$+ \dagger$ Nathan S. Lewis,,$\dagger$ Thomas F. Jaramillo,,$+\dagger$ Karen Chan, ${ }^{*,+, \dagger}$ and Jens K. Nørskov ${ }^{*,+, \dagger}$

$\dagger$ Joint Center for Artificial Photosynthesis $\ddagger S U N C A T$ Center for Interface Science and Catalysis, Department of Chemical Engineering, Stanford University, Stanford, California 94305 SLAC National Accelerator Laboratory, Menlo Park, California 94025

I Division of Chemistry and Chemical Engineering, California Institute of Technology, Pasadena, California 91125, United States

E-mail: chank@slac.stanford.edu; norskov@stanford.edu

\begin{abstract}
Bimetallic catalysts are promising for the most difficult thermal and electrochemical reactions but modeling the many diverse active sites on polycrystalline samples is an open challenge. We present a general framework for addressing this complexity in a systematic and predictive fashion. Active sites for every stable low-index facet of a bimetallic crystal are enumerated and cataloged yielding hundreds of possible active
\end{abstract}


sites. The activity of these sites is explored in parallel using a neural-network based surrogate model to share information between the many Density Functional Theory (DFT) relaxations, resulting in activity estimates with an order of magnitude fewer explicit DFT calculations. Sites with interesting activity were found and provide targets for follow-up calculations. This process was applied to the electrochemical reduction of $\mathrm{CO}_{2}$ on nickel gallium bimetallics and indicated that most facets had similar activity to Ni surfaces, but a few exposed Ni sites with a very favorable on-top CO configuration. This motif emerged naturally from the predictive modeling and represents a class of intermetallic $\mathrm{CO}_{2}$ reduction catalysts. These sites rationalize recent experimental reports of nickel gallium activity and why previous materials screens missed this exciting material. Most importantly these methods suggest that bimetallic catalysts will be discovered by studying facet reactivity and diversity of active sites more systematically.

\section{Keywords}

catalysis, electrochemistry, CO2 reduction, machine learning, DFT, energy 


\section{Introduction}

Intermetallic catalysts remain promising for tuning reactivity and developing new active site motifs with multiple functional adsorption sites but modeling these materials requires several orders of magnitude more calculations than modeling single transition metal surfaces. This complexity arises from two fundamental challenges in intermetallics: they can present more facets and terminations and they have far more feasible adsorption configurations, both due to a reduction in symmetry. In face of this complexity, computational chemists often consider only bimetallic versions of typical transition metal facets (e.g. (111), (100), or (211) surfaces), or simple schemes such as overlayers or islands. ${ }^{1,2}$ This problem is even more pronounced in theoretical screens for materials discovery, where resources per bimetallic are so limited that often a single facet and configuration is chosen. ${ }^{3}$ Unfortunately, these simplifications can lead to incorrect conclusions about the activity of a bimetallic catalyst or the active sites responsible for experimental activity. Even more troublesome is that promising new bimetallic active site motifs remain hidden in a sea of un-studied facets and active sites.

In this work, we show that bimetallic nanoparticles expose a much larger range of active site motifs than are typically considered in theoretical studies and present machine-learning methods that can efficiently address this combinatorial complexity. As an example we study $\mathrm{CO}_{2}$ reduction on nickel gallium intermetallics, as they have been shown to produce appreciable amounts of $\mathrm{C} 1$ and $\mathrm{C} 2$ products and are one of the few electrochemical cathode surfaces besides copper to do so. ${ }^{4}$ First, we show that recent progress in identifying stable crystal structures and enumerating possible facets and adsorption sites exposes a number of interesting active sites that would not appear in theoretical studies of simple (111) and (211) facets. Next, we report surface energy calculations that imply a markedly different facet distribution than would be expected for single metal nanoparticles and imply that these surfaces contain hundreds of chemically distinct active sites that must be considered. We describe a new approach using machine-learning neural network potentials to directly model the adsorption energy of $\mathrm{CO}$ on each site efficiently with a drastic reduction in the 
number of required DFT calculations. These methods rapidly predict the facets with the most interesting active sites and suggest active site motifs for further consideration. To illustrate this approach, we present a systematic study of bimetallic nickel gallium surfaces and show that the most promising active site motifs are isolated nickel atoms with surrounding gallium atoms. More detailed electrochemical kinetic studies demonstrate that this motif actually outperforms the typical linear scaling relations for close-packed surfaces and suggest a new approach to designing active materials. Importantly, this active site fell out of the systematic study and was automatically discovered, without requiring human intervention to design a new motif. These results suggest a path forward to the automated identification of single-site scaling relations and the systematic and predictive modeling of bimetallic facet reactivity.

Figure 1 shows the enormous complexity of the problem of $\mathrm{CO}_{2}$ reduction activity on nickel gallium intermetallic catalysts. The phase diagram shows that several bulk compositions are formed and stable at reducing potentials, including $\mathrm{Ni}, \mathrm{NiGa}, \mathrm{Ni}_{5} \mathrm{Ga}_{3}$ and $\mathrm{Ni}_{3} \mathrm{Ga}^{4}$ These four bulk structures have dozens of exposed facets, and these facets have hundreds of unique adsorption sites, according to the coordination of the adsorption site. Electrochemical $\mathrm{CO}_{2}$ reduction on these surfaces is a complex problem, so we adopt a simplified model developed from our understanding of single transition metal catalysts.

Recent mechanistic studies of $\mathrm{CO}_{2}$ reduction on copper and other transition metal surfaces have revealed two key challenges that must be overcome to generate appreciable amounts of higher hydrocarbon or alcohol products. The first challenge is that the ratelimiting protonation of $\mathrm{CO}$ to $\mathrm{CHO}$ must be made feasible, with both a favorable active site and a favorable transition state energy. For materials that follow the scaling behaviour of pure transition metals, the CO binding energy is the best current descriptor for the barrier of the CO protonation step, with weaker CO binding materials having lower barriers. However, materials with a positive $\mathrm{CO}$ binding free energy would desorb $\mathrm{CO}$ as a final product rather than make higher products. Second, the hydrogen evolution reaction (HER) must also be 


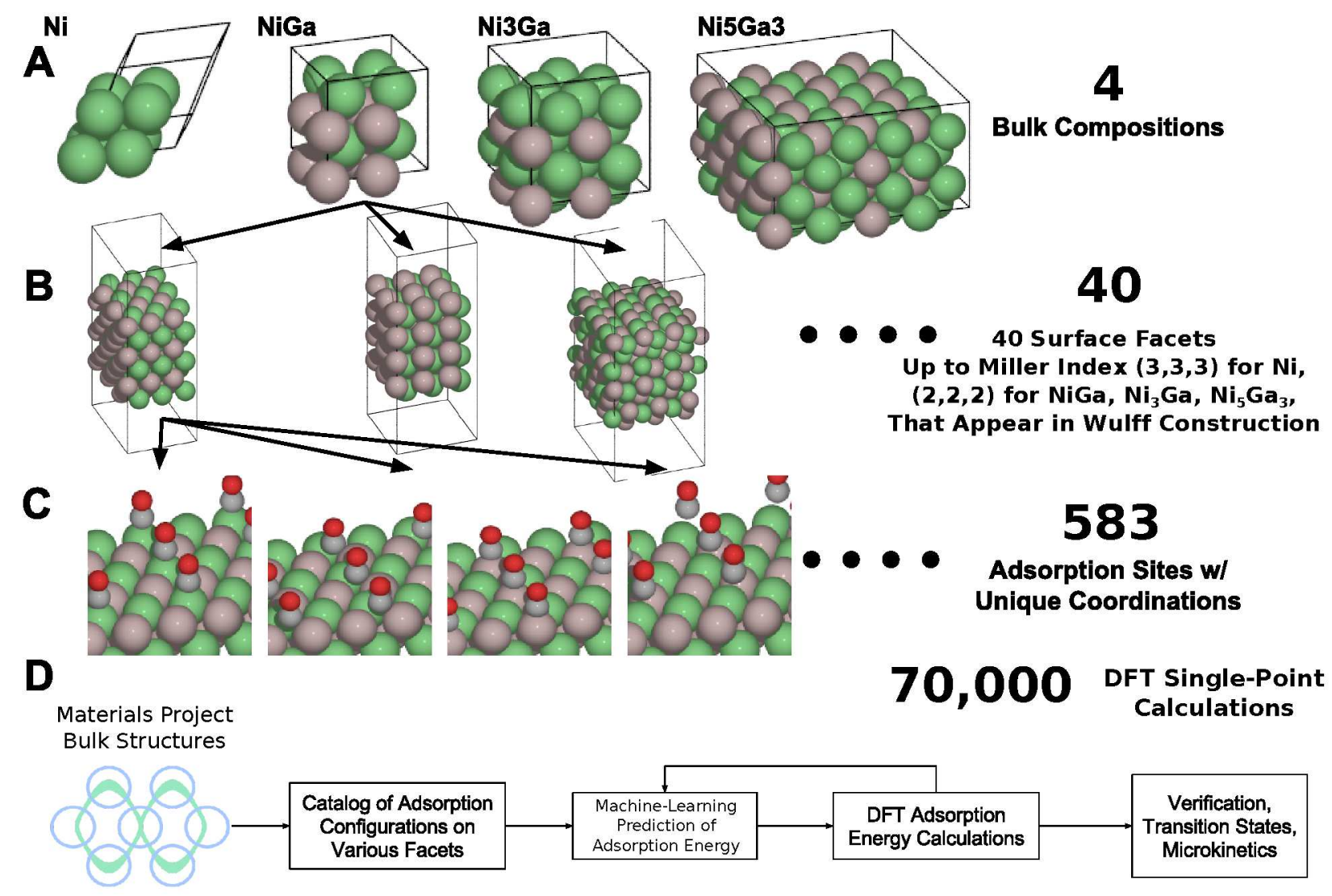

Figure 1: Combinatorial challenge of identifying active sites and surfaces for bimetallic catalysts A) Four Ni/Ga intermetallics made experimentally and identified as the lower hull by the Materials Project. B) 40 identified facets/terminations, up to Miller index $(3,3,3)$. Facets often expose two asymmetric terminations so much be considered separately. C) 583 adsorption configurations identified with unique average coordination of bonding metal atoms. D) High-throughput methodology developed to catalog and rapidly evaluate necessary thermodynamic quantities for this combinatorial problem. 


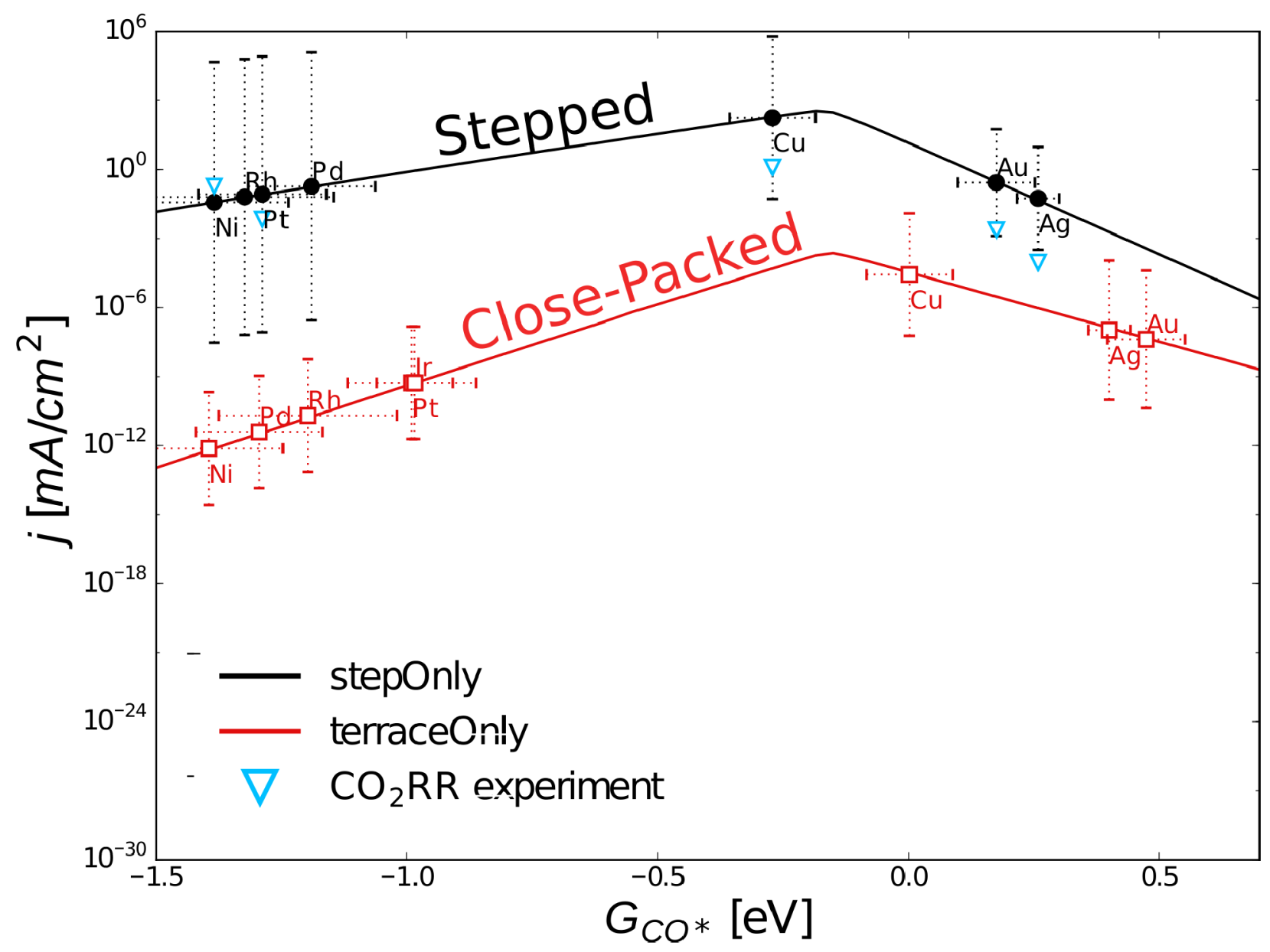

Figure 2: CO adsorption free energy describes activity for metal surfaces. The predicted rate towards $\mathrm{CO}$ reduction products from a microkinetic model ${ }^{5}$ is shown in solid lines for both terrace (111) and step (211) configurations as a function of the surface CO binding free energy $G_{C O}$. Uniformly the rate for the step sites are higher. Both site types show a maximum in activity for weak-binding $\mathrm{CO}$ surfaces. Copper, the best single-metal catalyst for the production of $\mathrm{C} 1$ and $\mathrm{C} 2$ products, is near the top of these trends. This detailed model suggests that the search for electrochemical $\mathrm{CO}_{2}$ reduction catalysts should focus on finding weak-binding CO materials. 


\section{Theoretical Methods}

\section{Enumeration and Prediction of Stable Facets and Adsorption Sites}

Determining facet stability and reconstruction for bimetallic catalysts is an open question, especially in the presence of solvation and strongly binding adsorbates. There is no general method to quickly determine the surface area or facet distribution of sites for an experimental polycrystalline catalyst. We generated a catalog of all nickel gallium facets up to a maximum Miller index of $(2,2,2)$, and all nickel facets up to $(3,3,3)$. Facets were generated using the python package pymatgen. ${ }^{6}$ All generated facets were bulk-terminated to reduce the number of surfaces to consider. Using this reduction technique, 176 unique surfaces were identified for the four compositions ( $\left.\mathrm{Ni}, \mathrm{NiGa}, \mathrm{Ni}_{3} \mathrm{Ga}, \mathrm{Ni}_{5} \mathrm{Ga}_{3}\right)$. Effects such as adsorbate-induced segregation or surface reconstruction were not considered.

An established method ${ }^{7}$ for determining surface energy using DFT energies from slabs of three different thicknesses was used to generate likely facet distributions for each of the bimetallic compositions as illustrated in Figure 3. Surface energies were calculated for every surface in the catalog described above (including Ni surfaces up to Miller index $(3,3,3)$ and every Ni/Ga bimetallic up to Miller index $(2,2,2))$. By extrapolating the bulk energy from 

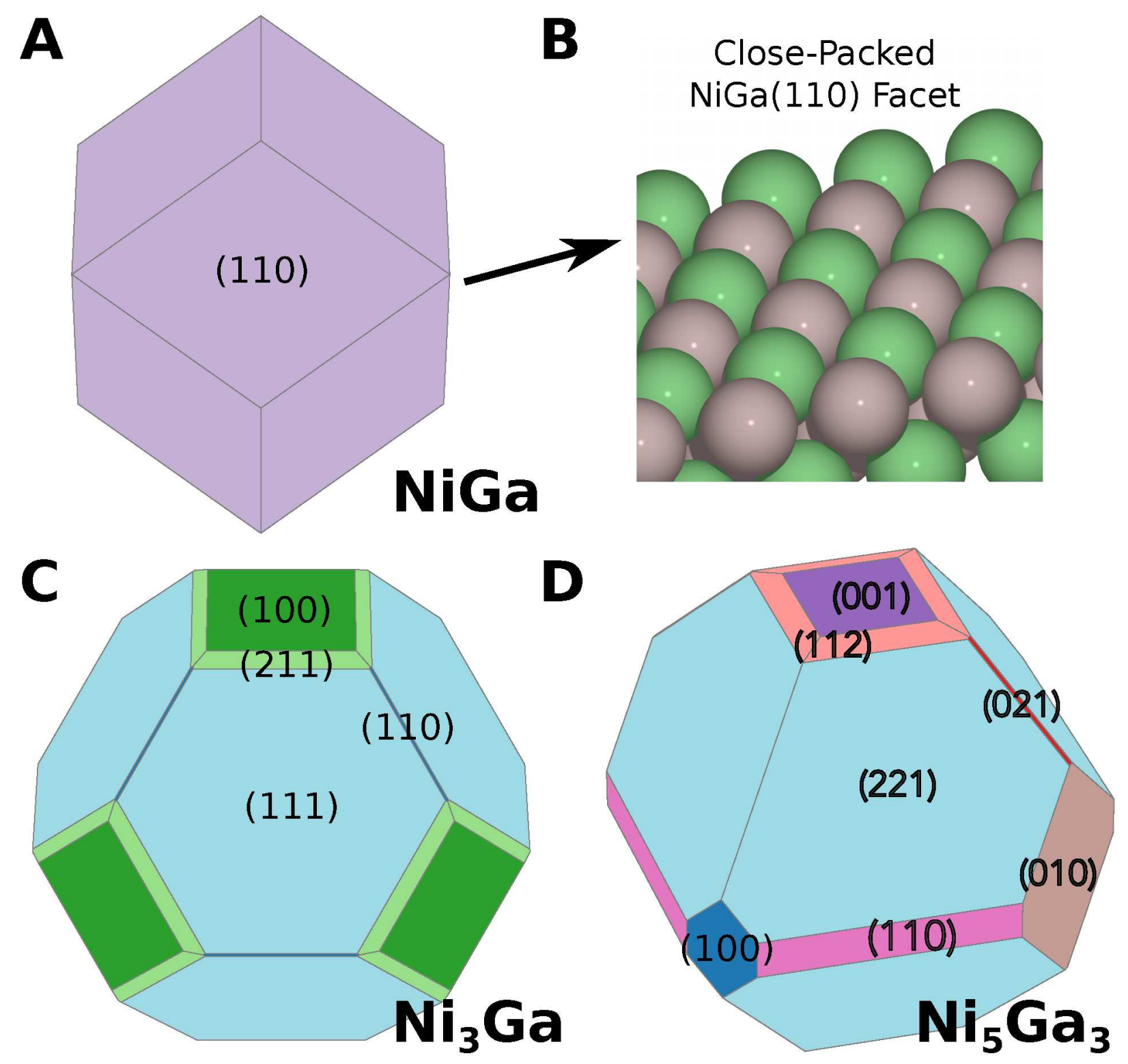

Figure 3: Bimetallic alloys present significantly different facets than those used typical model facets $[(100),(111),(211)]$ Wullf crystal reconstructions showing equilibrium nanoparticle shapes according to calculated facet surface energies. All three bimetallic alloys present remarkably different facet distributions, suggesting simple (111),(100), (211) screens are insufficient for predicting bimetallic activity. A) Wulff crystal for NiGa bimetallic, which is dominated by (110) facets. B) Image of the close-packed (110) NiGa facet with alternating $\mathrm{Ni}$ and Ga atoms on the surface. C) Wulff crystal for Ni3Ga, which present arange of surfaces. D) Wulff crystal for Ni5Ga3, which presents more step surfaces than NiGa or Ni3Ga. 
the successive surface calculations, surface energies were obtained from slab energies at three different thicknesses. Details of the DFT methods used are included in the Supplementary Information. Surface energy calculations for Ni facets were in agreement with previous theoretical work, ${ }^{8}$ as well as experimental characterization of $\mathrm{Ni}$ nanoparticles. ${ }^{9}$ Wulff crystal constructions were used to estimate available surface areas and indicate likelihood of experimental occurrence. We note that this approach may not show quantitative agreement due to solvent effects or nanoparticle-induced compressive strain, but qualitatively the approach should yield the most likely facets to appear in polycrystalline samples. All facets appearing the Wulff constructions for each composition were included for adsorption energy analysis. The precise quantitative area of each facet from the Wulff construction was not needed. This process resulted in just a single facet for NiGa, the (110) facet, so several additional NiGa facets were included that included step sites. This process resulted in a total list of 23 Miller indices to be considered in this study. Due to slab asymmetries, these 23 Miller index slabs yielded 40 unique surfaces to consider. Interestingly the $\mathrm{Ni}_{3} \mathrm{Ga}$ and $\mathrm{Ni}_{5} \mathrm{Ga}_{3}$ Wulff construction contained a large number of facets with substantial surface area, in contrast to the case of NiGa which contains almost entirely the close packed (110) facet. This result reinforces that the typical facets used in single metal studies including (100), (111), and (211), may not be indicative of the facets in bimetallic systems.

Asymmetric facet terminations were also included as possible active sites. For Ni surfaces, the cuts were symmetric and the top and bottom surfaces of the slabs were considered. For the bimetallic crystals, some cuts were necessarily asymmetric, for example the NiGa(100) facet. This facet exposes alternating layers of nickel and gallium sheets, hence facets with stoichimetric ratios of the bulk would have one side of the slab with a gallium termination, and the other with a nickel termination. In physical systems, one termination would likely be preferential and could be formed by adding a non-stoichiometric layer. Identifying preferential surface energies of various facet terminations under electrochemical conditions with the possibility of plating or reconstruction is an open question with interesting thermody- 
namic considerations, for example, a solution-phase $\mathrm{Ni}$ or Ga chemical potential would be required to determine if one of the two asymmetric surfaces would plate to become symmetric. Therefore we simply included both possible terminations; the reported surface energy corresponds to the average of both terminations (the asymmetric top and bottom sides of the stoichiometric slabs). Decomposing these surface energies into separate energies for each side is an open question for bimetallics and should lead to more accurate surface energies in the future.

Adsorption sites for the most likely facets were enumerated to create a catalog of all possible adsorption sites. The enumeration was made possible by recent extension to the Materials Project library pymatgen. ${ }^{10}$ The number of adsorption sites was typically much larger for bimetallic surfaces than for single metal surfaces, due to reductions in symmetry. Each adsorption site was characterized by coordination, including the number of Ni neighbors, the number of Ga neighbors, the average $\mathrm{Ni}$ and $\mathrm{Ga}$ coordination of neighboring $\mathrm{Ni}$ atoms, the average $\mathrm{Ni}$ and Ga coordination of neighboring Ga atoms, and the fraction of the $\mathrm{Ni}$ in the alloy, for a total of 7 descriptors

$$
\text { [\#Ni, \#Ga, Avg.\#Ni-Ni, Avg.\#Ni-Ga, Avg.\#Ga-Ni, Avg.\#Ga-Ga, Bulk Fraction]. }
$$

In this way, the type of site could be inferred (e.g. on-top Ni vs Ni-Ga bridge), and similar sites with different coordination (e.g. Ni-Ni bridge on a terrace vs Ni-Ni bridge on a step) could also be identified. Only sites with unique descriptions were kept, so that multiple identical sites were not considered on a surface. Developing a generalized coordination number to handle this more generally is a topic of interest in the literature. ${ }^{11,12}$

\section{Machine Learning Prediction of Adsorption Energies}

DFT-fitted neural network potentials were used to greatly reduce the many thousands of DFT calculations required to obtain relaxed adsorption energies for each adsorption site on 
every facet. All-DFT relaxations often take 20-100 steps in a local minimizer with each step requiring a full single-point calculation. Performing DFT relaxations for every adsorption site is inefficient due to a lack of information sharing between relaxations. Also, every step in every relaxation is an independent DFT calculation with little information from previous steps and no information from relaxations at similar sites. Using a neural network potential allows information to be shared and the simulation process accelerated, as has been demonstrated for the reactivity on metal surfaces, ${ }^{13}$ studying the solvation of alloy nanoparticles, ${ }^{14}$ and for accelerating the study of alloy segregation, ${ }^{15}$ among others. In this work we use a set of neural network potentials were used to simultaneously relax all possible adsorption sites, select configurations to study with DFT, add to the training set, and refine.

Neural network potentials were used to perform relaxations of adsorptions and direct predictions of adsorption energy using only information from near-adsorbate atoms. The per-atom neural network potentials were similar to a standard scheme shown to be effective in recent literature ${ }^{16}$ and implemented recently in an open-source software package, ${ }^{17}$ one of several implementations in the literature. ${ }^{18,19}$ However, rather than predict the electronic energy reported by the DFT code as is standard for these methods, the adsorption energy was instead chosen as a target, which had several advantages. First, the adsorption energy is a small well-normalized energy, usually ranging from $-3 \mathrm{eV}$ to $+1 \mathrm{eV}$, so that energy normalization was not a problem. Second, this scheme only requires a relaxation of the adsorbate and slab to predict the adsorption energy using the surrogate model, rather than a prediction for the slab+adsorbate and bare slab and relying on cancellation of energies as is normal in DFT. A relaxation for the bare slab was only necessary to get the small relaxation energy of the bare slab as the top layer was relaxed. Finally, directly predicting the adsorption energy allowed for a significant reduction in system size, because only nearadsorbate metallic atoms contribute to the adsorption energy, so atoms deep in the bulk can be neglected.

The precise adsorption energy prediction scheme is illustrated in Figure 4. A standard 

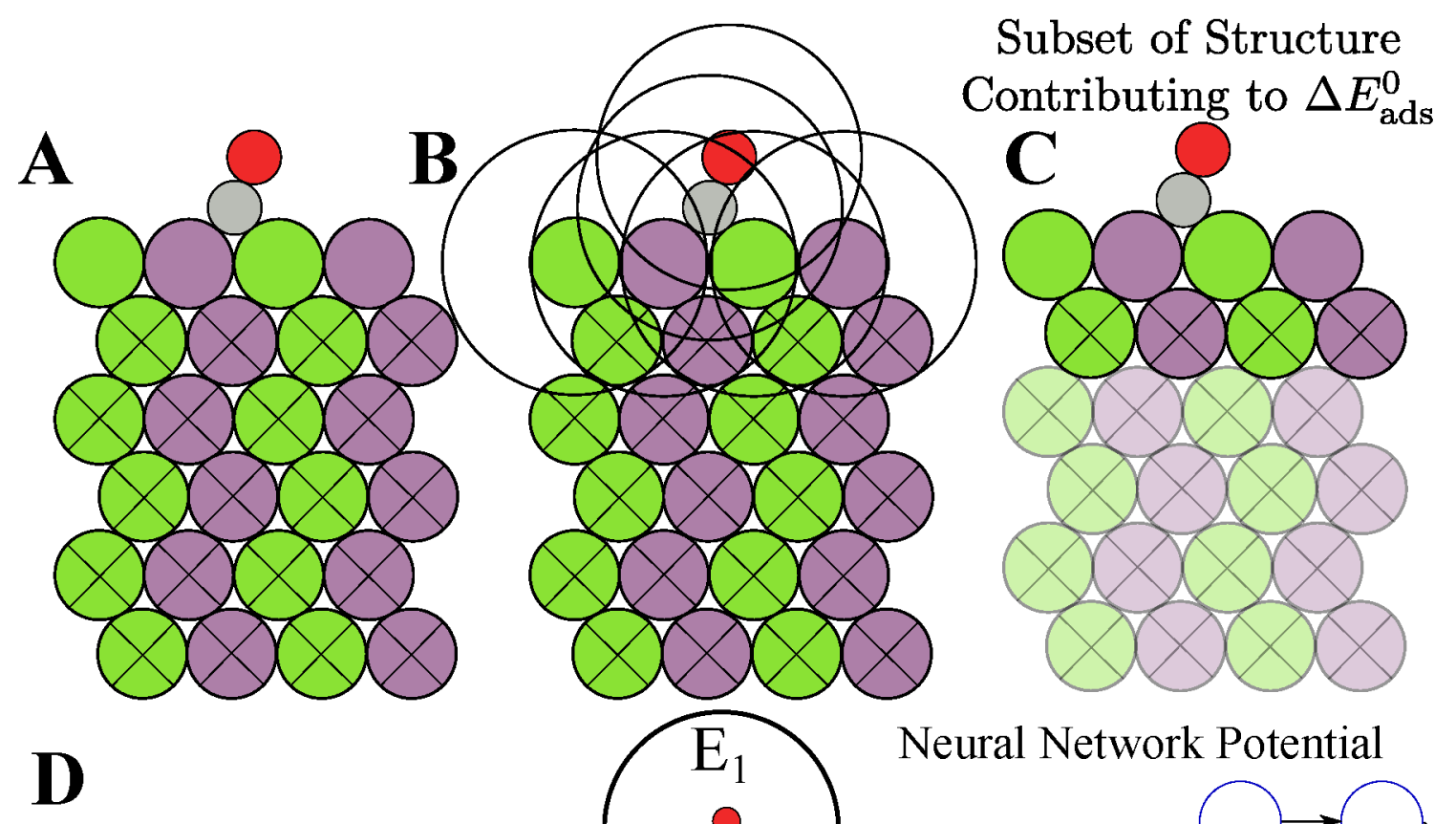

\section{.}

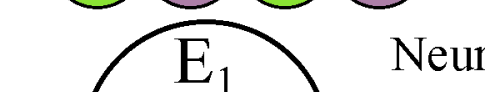

Neural Network Potential

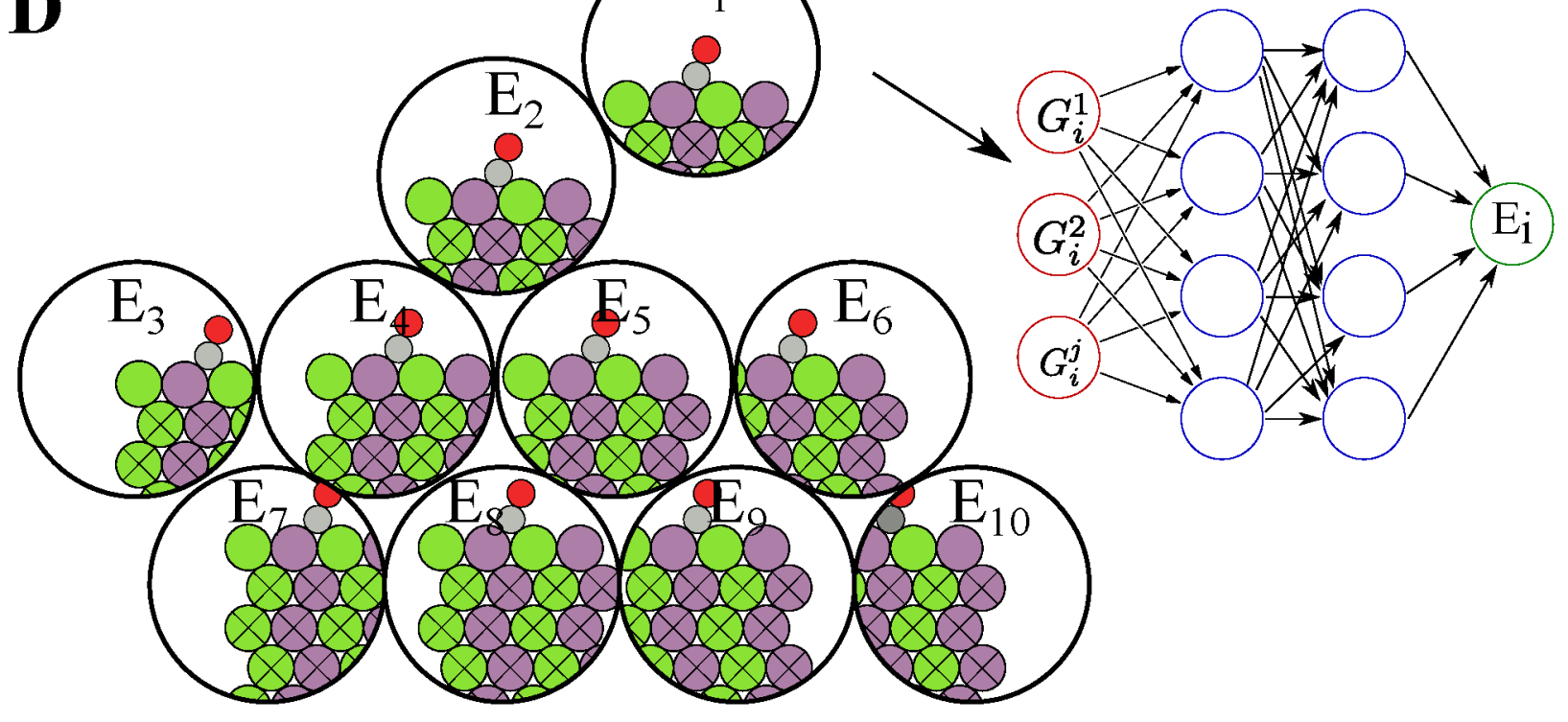

$$
\Delta E_{\mathrm{ads}}^{0}=E_{\text {slab }+ \text { ads }}-E_{\mathrm{slab}}^{\text {unrelaxed }}-E_{\text {gas }}=\sum_{i} E_{i}
$$

Figure 4: Cartoon of the neural network potential used to directly relax and predict adsorption energies for small molecules. (A) All-atom representation of a typical bimetallic surface with a $\mathrm{CO}$ adsorbate, with the top layer free to relax. (B) Identification of all atoms near those with degrees freedom to include in the reduced representation. (C) Subset of the structure which is used to predict the adsorption energy. (D) The local region around each atom is used to generate a geometric fingerprint, which is fed through a neural network to provide an atomic contribution to the adsorption energy. The predicted adsorption energy is a summation over these atomic contributions. 
surface adsorbate model was used and represented the configuration that the underlying DFT code would see when asked for electronic energies. For predictions of the adsorption energy, all atoms within $3.5 \AA$ of unconstrained atoms (those allowed to relax, generally the adsorbate or the top layer) was chosen for inclusion in the final energy representation, so that forces on these atoms could be properly reconciled. Each atom in this reduced representation was then fingerprinted and its energy predicted with a neural network specific to the atomic species (Ni, Ga, C, H, O). The energy for training was the adsorption energy relative to the unrelaxed slab and the gas-phase CO energy, $\Delta E_{a d s}^{0}=E_{\text {slab+ads }}-E_{\text {slab }}^{\text {unrelaxed }}-E_{\text {gas }}$. This process requires only one single-point DFT calculation for the unrelaxed slab and a relaxed gas energy to calculate the adsorption energy for any given snapshot of a slab and adsorbate. A similar prediction was made for the bare slab so that the relative energy of the relaxed slab with respect to the unrelaxed slab could be calculated as $\Delta E^{0}=E_{\text {slab }}-E_{\text {slab }}^{\text {unrelaxed }}$. The final adsorption energy was thus $\Delta E_{a d s}=\Delta E_{a d s}^{0}-\Delta E^{0}=E_{\text {slab+ads }}-E_{\text {slab }}-E_{\text {gas }}$. In most cases the bare slabs were found to relax little and $\Delta E^{0}$ was usually under $0.1 \mathrm{eV}$. The final predicted free energy was calculated by applying a standard free energy correction for CO as described in the methods section. This process allows the adsorbate to be relaxed using only fingerprints of the near-adsorbate region of the slab.

Efficiently training large neural network potentials with thousands of single point calculations is an open challenge, especially for large systems in the range of this example. The machine learning community has made rapid progress in developing codes and methods to make this process more efficient, from fast implementations of neural networks, to improved optimizers, and techniques such as dropout ${ }^{20}$ and L1/L2 regression to reduce problems associated with overfitting. To take advantage of these lessons, we developed and contributed a new implementation of neural network potentials based on the Google-supported tensorflow library. ${ }^{21}$ This implementation resulted in greater than a 10-fold increase in training rate and allowed for more sophisticated training of potentials, making this approach practical for the problems addressed herein. Further, error estimates for the surrogate model were tracked 
by monitoring the stochastic distribution of predictions under neural network dropout. ${ }^{22}$ Details of the training scheme are included in the Supporting Information and the code is now implemented in the open source AMP package. ${ }^{17}$

\section{On-line Model Refinement}

An iterative process, illustrated in Figure 5, was used to train the surrogate model, relax adsorbates according to the surrogate model, and select configurations to study with singlepoint DFT calculations to add to the training set. This process was bootstrapped starting with a single DFT relaxation of a CO molecule on an arbitrary nickel gallium surface. The relaxations using the surrogate model were carried out in parallel and resulted in a predicted $\mathrm{CO}$ adsorption energy for each of the sites outlined above (583 relaxations, for a total of 70,000 single-point calculations). The model energy uncertainty was tracked along each relaxation. The relaxation was halted if the uncertainty rose above $0.2 \mathrm{eV}$. Adsorption configurations selected for refinement were automatically submitted for calculation using the fireworks workflow manager as a single-point DFT calculation. ${ }^{23}$

To verify the convergence of this surrogate model approach for predicting adsorption energies, standard DFT relaxations were used to get the adsorption energy for each adsorption site in this work. This process resulted in approximately 70,000 DFT single-point calculations and represented approximately 50,000 core-hours of computational time. This approach was feasible for this study, but the number of DFT calculations would rapidly increase if more facets or adsorbates were included. However, the number of DFT single-point calculations needed to train the machine-learning would not scale in the same fashion due to the similarity of sites on different facets.

The convergence of the surrogate model to DFT predictions is illustrated in Figure 5(B,C). With just one DFT relaxation as a starting point, reported adsorption energies are clustered around 0 eV. After approximately 2000 DFT single-points have been added, a correlation begins to form, and after 4000 DFT single-points there is a strong correlation 

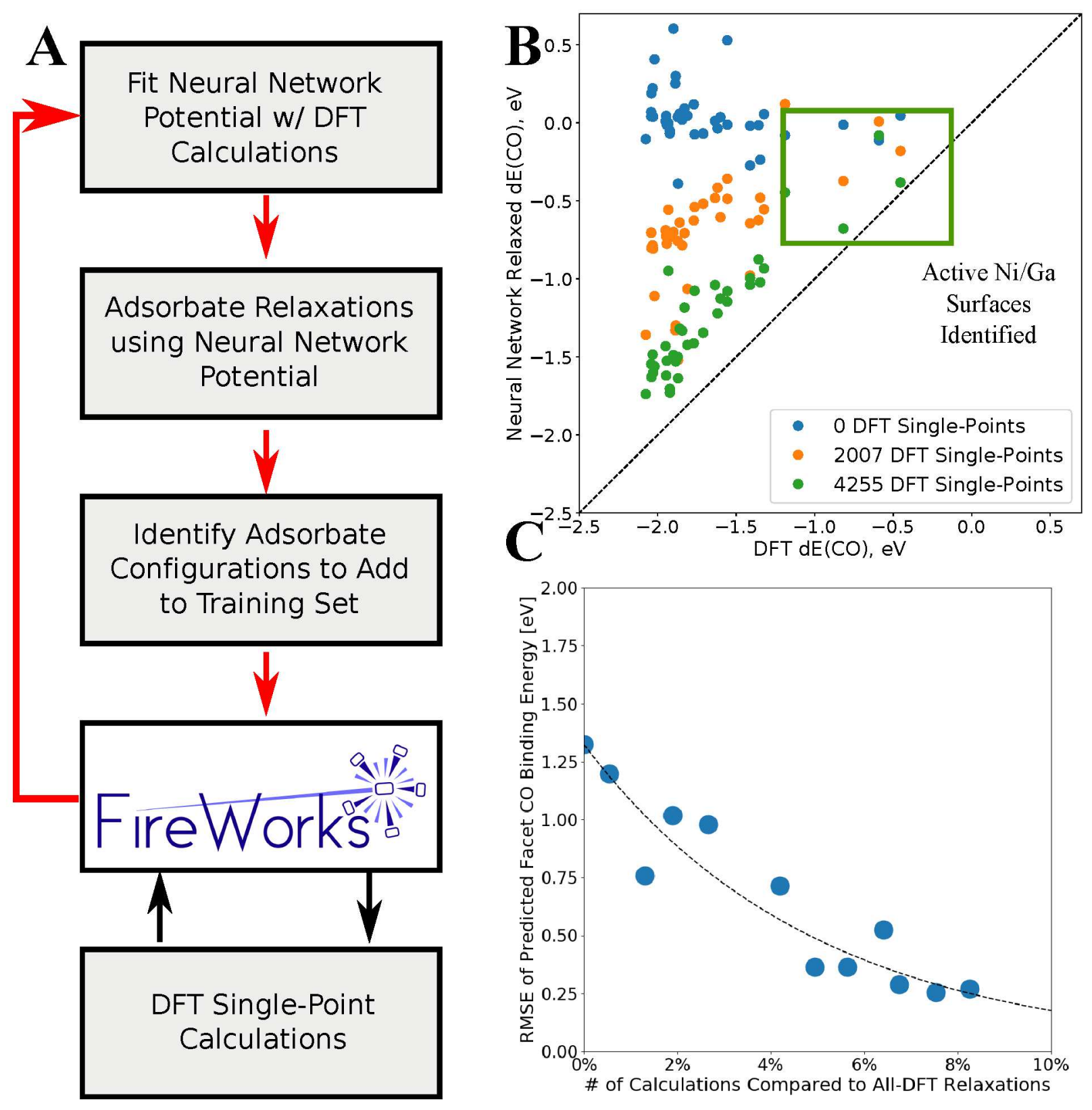

Figure 5: Convergence of facet $\mathrm{CO}$ adsorption energies for the machine learning model. (A) Scheme used for cyclical training and application of the model, and acquisition of new training data via DFT single-point calculations. (B) Parity plot for several iterations of the convergence system, starting from very poor predictions and converging to more accurate predictions of adsorption energy. After just a few thousand single-point calculations, the surfaces with the most interesting $\mathrm{CO}$ adsorption energies have been isolated, showing that qualitative accuracy is higher than quantitative accuracy would suggest. (C) Convergence of the accuracy of the $\mathrm{CO}$ adsorption energies with respect to the training set size. Points are stochastic since the neural network training algorithms are stochastic. 
between the reported adsorption energy for each facet and the DFT calculated values. The RMSE error converges steadily with each iteration of this process, as shown in Figure 5C, starting at well over $1 \mathrm{eV}$ RMSE with nearly no information and approaching DFT accuracy of $0.2 \mathrm{eV}$. This result required only $10 \%$ of DFT calculations required in a full explicit DFT study.

Configurations were selected for refinement by performing relaxations on all $583 \mathrm{CO}$ adsorption sites simultaneously using the surrogate model. The first step in each relaxation with an estimated error above $0.2 \mathrm{eV}$ was selected for refinement. If all steps in the relaxation had estimated errors below $0.2 \mathrm{eV}$, the final point was selected for refinement if the uncertainty was over $0.1 \mathrm{eV}$. In this way, several hundred configurations were added to the training set with a DFT single-point calculation at each iteration with a very modest computational cost.

\section{Results/Discussion}

\section{Bimetallic Facet Reactivity}

The CO adsorption energies for each facet calculated above were used to predict the activity and selectivity of each facet based on linear scaling relations and microkinetic model developed from previous studies on single metal transition metal (111) and (211) facets. In the microkinetic model, all free energies and kinetic barriers are assumed to be linearly dependent on either the $\mathrm{CO}$ adsorption energy or the transition state energy of the $\mathrm{CO}$ to $\mathrm{CHO}$ protonation step. ${ }^{5}$ This allows the activity of a material to be predicted solely based on these two values, as illustrated in Figure 6. Typical scaling relations for the transition state energy vs the CO adsorption energy are also shown for terrace and step active sites. Assuming surfaces fall on one of these two scaling lines, it is possible to estimate the hydrocarbon production rate with just the type of facet (terrace vs step) and the CO adsorption energy. Various nickel gallium facets are thus included by interpolating onto the scaling lines. Most 

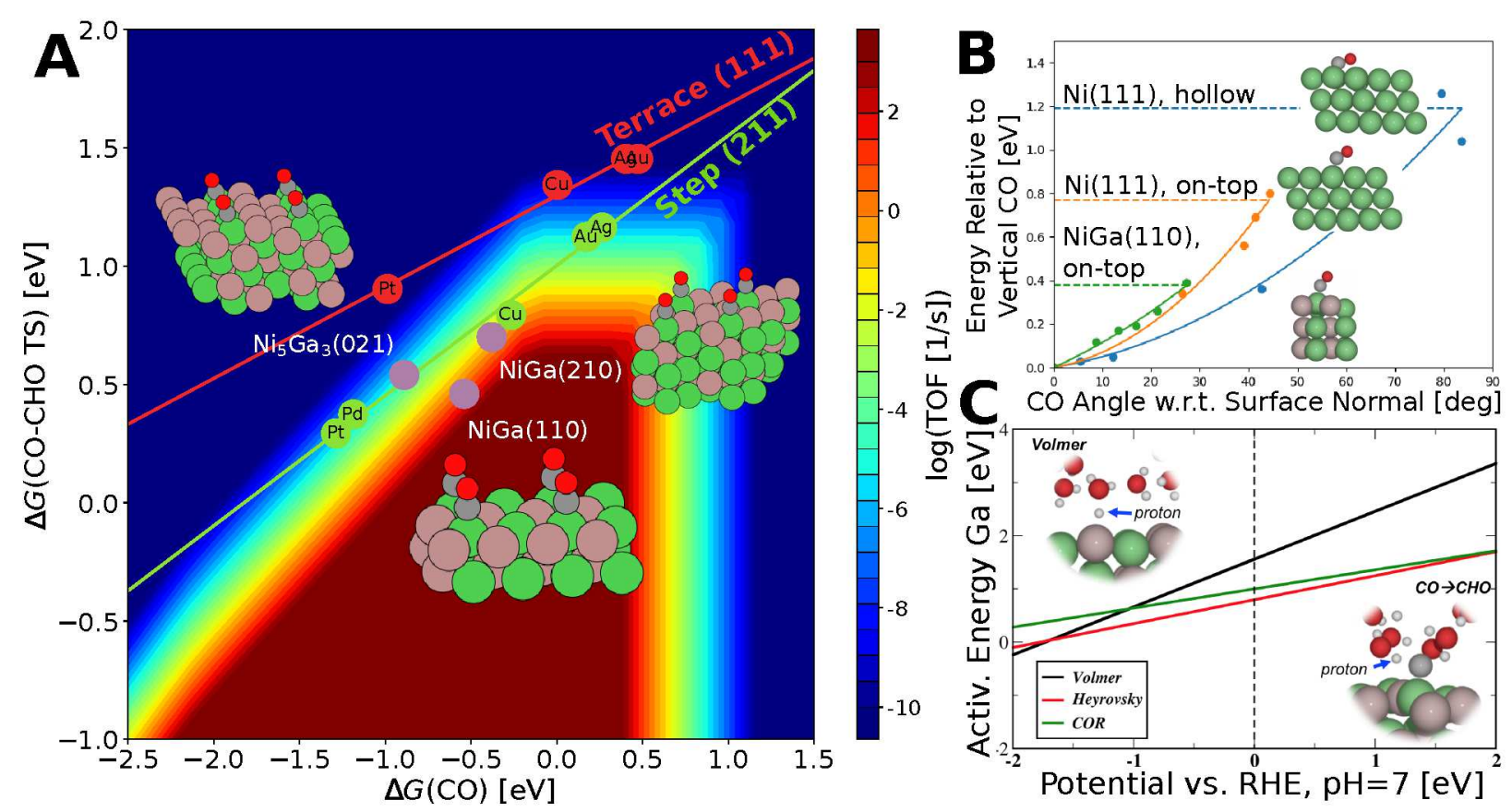

Figure 6: Predicted activity from microkinetic model of transition metal and nickel gallium facets. (A) Solid green and red circles are DFT calculated transition state energies of singlemetal surfaces for either terrace or step surfaces. Purple circles are the three nickel gallium facets for which explicit transition state calculations were performed. All three explicit calculations show step-like scaling relations due to the on-top configuration of $\mathrm{CO}$ on these surfaces which more resembles the single-metal step configuration. (B) Rotation energy for $\mathrm{CO}$ on $\mathrm{Ni}$ and $\mathrm{NiGa(110)}$ sites, from straight up to the surface-C-O angle for $\mathrm{CHO}$. (C) Hydrogen selectivity problem, showing that at all applied potentials the kinetic barrier for hydrogen evolution is lower than (but comparable to) the barrier for CO reduction. 
of the nickel gallium adsorption sites have similar adsorption energies to the pure Ni facets since the CO adsorption energy is dominated by the availability of 2-fold, 3-fold and 4-fold $\mathrm{Ni}$ sites. Predictions of the selectivity and coverage from the microkinetic model are also included in the Supporting Information.

Surprisingly, several nickel gallium facets have CO binding energies very close to the best possible rates for materials that follow the existing terrace or step scaling relations, including $\mathrm{NiGa}(210), \mathrm{NiGa}(110)$, and $\mathrm{Ni}_{5} \mathrm{Ga}_{3}(021)$. All of these facets expose $\mathrm{Ni}$ active sites with no nearby $\mathrm{Ni}$ sites to form higher coordination adsorption sites (bridge, 3-fold, etc). We note that all the surfaces were quickly predicted to have interesting adsorption energies by the machine-learning algorithm above. Given the interesting nature of these adsorption sites, predicted with our ML methods and confirmed with DFT relaxations, we explicitly calculated the transition state of the electrochemical CO protonation explicitly. The interesting nature of these sites were confirmed with explicit solvent transition state calculations.

\section{Electrochemical Kinetics of CO Protonation}

Transition state calculations were completed for CO protonation on the most active surfaces as calculated above, $\mathrm{NiGa}(210), \mathrm{NiGa}(110)$, and $\mathrm{Ni}_{5} \mathrm{Ga}_{3}(021)$. Calculations were completed with one layer of explicit solvent, and were corrected to a constant potential using a recently developed charge extrapolation scheme. ${ }^{24}$ These explicit calculations are included in Figure $6 \mathrm{~A}$ as filled circles. The scaling lines for both stepped (211) and terrace (111) sites for transition metal surfaces are shown, with stepped surfaces performing significantly better due to their lower activation energy for CO protonation. ${ }^{5}$ All three nickel gallium facets appear to follow the step scaling relation, even though $\mathrm{NiGa}(110)$ for instance corresponds to the closest-packed bcc facet.

The surprisingly facile activation energies on these nickel gallium surfaces can be explained by the on-top nature of the active site which makes rotation of the $\mathrm{CO}$ to the transition state easier. Figure $6 \mathrm{~B}$ shows the rotation energy of $\mathrm{CO}$ for the 3 -fold and on- 
top sites of $\mathrm{Ni}(111)$ and the $\mathrm{NiGa}(110)$ facet; even on $\mathrm{Ni}(111)$, the on-top site has a more facile energy of rotation than the 3-fold site; however this would not be reflected in the CO reduction rate of $\mathrm{Ni}(111)$ since $\mathrm{CO}$ adsorbs much more strongly on the 3 -fold site.

We studied the activation energies of the hydrogen evolution reaction for these surfaces as illustrated in Figure 6C. The Heyrovsky and Volmer processes of HER reactions were both considered, and the potential-dependent transition state energy for each are included. The Heyrovsky transition state energy was predicted to have the lowest barrier in the range of potentials relevant to the $\mathrm{CO}_{2}$ reduction reaction. Therefore, the Heyrovsky process on $\mathrm{NiGa}(110)$ will be always faster than $\mathrm{CO}$ protonation and $\mathrm{H}$ adsorption will not be a competing factor. The interesting finding is the Volmer process competes with CO protonation, crossing at about $-1 \mathrm{~V}$ vs. RHE. Below this potential, both HER processes have a lower activation energy than $\mathrm{CO}$ protonation, consistent with the experimental observation that HER dominates at very reducing potentials. Since the transition state of Volmer process has more charge transferred, it has a larger slope vs potential than CO protonation. ${ }^{24}$ These barriers were input into a microkinetic model, ${ }^{5}$ shown in Figure $6 \mathrm{~A}$.

These theoretical results suggest that the experimentally observed activity of nickel gallium surfaces for electrochemical $\mathrm{CO}_{2}$ reaction ${ }^{4}$ can be qualitatively explained by $\mathrm{Ni}$ active sites surrounded by surface gallium atoms. The experimental activity is shown in Figure 7(A). First, the electrochemical reduction of $\mathrm{CO}_{2}$ is shown to have a lower onset potential for Ni/Ga bimetallics than for copper films. Second, at all potentials the selectivity to hydrocarbon or alchohol products is poor for $\mathrm{Ni} / \mathrm{Ga}$ bimetallics due to the lower kinetic barrier for HER than for CO reduction. Qualitatively, the same trend is suggested by the microkinetic model; leveling off in COR activity at negative overpotentials is not reproduced theoretically, however, surface segregation effects may be at play. The difference between the HER and the $\mathrm{CO}_{2}$ reduction barriers predicted to be nearly potential-independent, shown in Figure 6C, explaining why hydrogen selectivity is a problem for Ni/Ga bimetallics at all applied potentials. 

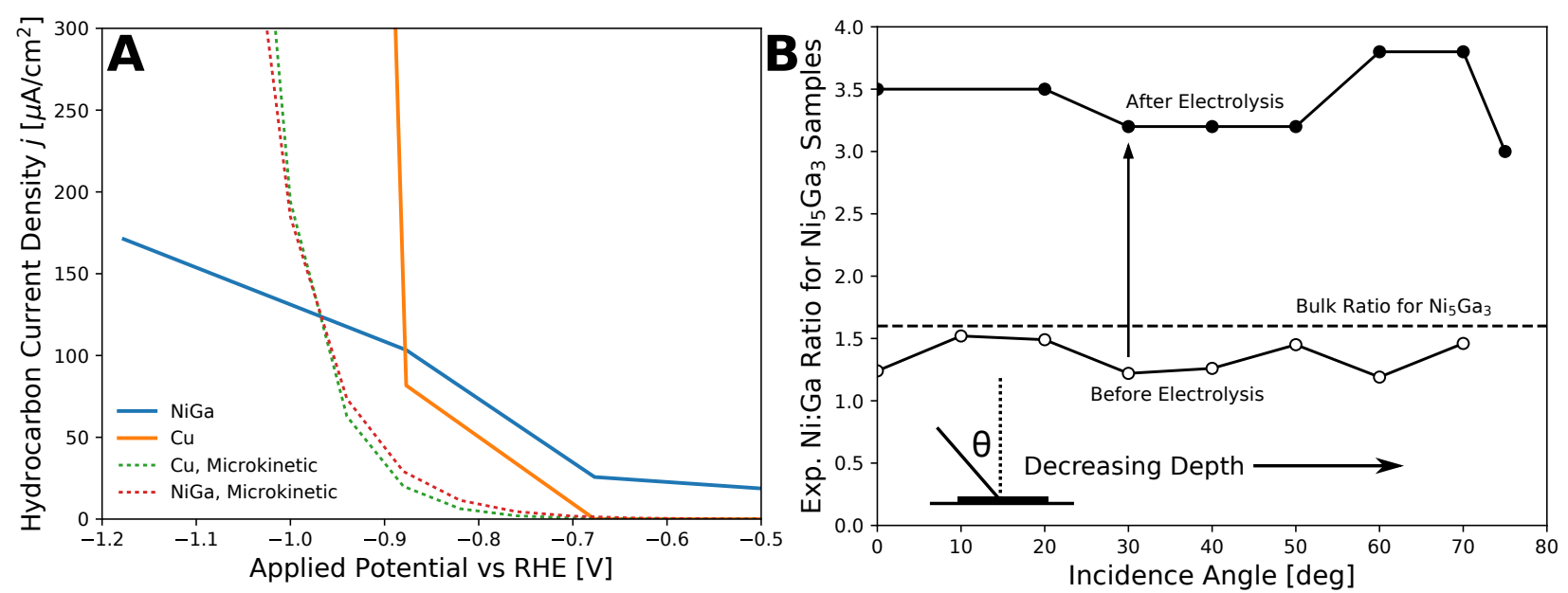

Figure 7: Experimental evidence for surface gallium impacting electrode performance. (A) Experimental performance of $\mathrm{Ni} / \mathrm{Ga}$ bimetallic catalysts for the electrochemical reduction $\mathrm{CO}_{2}$ to hydrocarbons ${ }^{4}$ compared to the microkinetic model in this work. The total current density to hydrocarbon and alcohol products is compared vs the applied potential. The onset potential for $\mathrm{CO}_{2}$ reduction is significantly more positive than the best known catalyst, $\mathrm{Cu}$, but $\mathrm{Ni} / \mathrm{Ga}$ intermetallics do not achieve the high selectivity of $\mathrm{Cu}$ at large applied potentials. The leveling of current past $-0.9 \mathrm{~V}$ is not captured in the microkinetic model, but thought to be continued $\mathrm{Ni}$ surface segregation. (B) Experimental evidence that a significant amount of surface gallium remains at large reducing potentials, despite the strong $\mathrm{CO}$ binding to $\mathrm{Ni}$ being a driving force for surface segregation. 
Spectroscopic investigations have confirmed that Ni and Ga remain on the surface after catalysis. Angle resolved X-ray photoelectron spectroscopic (ARXPS) measurements were performed on fresh samples and ones that had been polarized at $-1.5 \mathrm{~V}$ vs. $\mathrm{Ag} / \mathrm{AgCl}$ for 2 hr under 1 atm $\mathrm{CO}_{2}$, at which point over $60 \mathrm{C}$ of charge had been passed (details included in the Supplementary Information). Detailed spectra, included in Figure S1-S4, show both $\mathrm{Ni}$ and Ga present throughout the surface after long-term electrolyses, a summary of which is in Figure 7B. The approximate angle-resolved ratio is included in Figure 7B, showing a $\mathrm{Ni}$ richening of the surface after $\mathrm{CO}_{2}$ reduction as would be expected for a strong $\mathrm{CO}$ binding metal like Ni. However, the confirmed presence of surface Ga suggests that these minority sites are most likely responsible for the considerably different activity of these Ni/Ga intermetallics compared to pure Ni films.

\section{Conclusions}

Bimetallic nanoparticle catalysis is inherently challenging due to the tremendous heterogeneity of active sites exposed. The crystal structures vary with composition, the exposed facets are different from what normal single metal nanoparticles might demonstrate, and the heterogeneity of the surfaces requires many more DFT calculations to capture correctly. This complexity is not well served by the traditional approaches that have worked for developing understanding in single-metal catalysts. This work shows that this problem can be approached systematically with all active sites considered. The number of DFT calculations is very large but feasible for a small number of compositions. Using newly developed machine learning potentials as a surrogate model for DFT allows for an order of magnitude reduction in the number of DFT calculations required and makes this treatment feasible for every bimetallic of interest.

The systematic approach in this work led to the discovery of a previously unconsidered active site for Ni/Ga intermetallics - active Ni atoms surrounded by surface Ga atoms. 
These active sites demonstrate the best thermodynamics for CO reduction, but even more importantly exhibit step-like kinetic behavior. The discovery of this motif contributes greatly to our understanding of what makes a good $\mathrm{CO}_{2}$ reduction catalyst, we now know that it is not necessarily just stepped surfaces that account for observed activities. The insight and methods of this approach will allow similar new motifs to be cataloged and characterized for many other intermetallic compounds.

This approach does not currently consider surface segregation or significant disorder in the crystal composition, both of which are likely for intermetallics with small heats of formation and situations with large adsorption energies. The methods developed in this work should be equally applicable to such systems, but the surface generation procedures will have to extended to enumerate these new materials (overlayer structures, disordered materials, defects).

\section{Competing Financial Interests}

The authors declare no competing financial interests.

\section{Acknowledgment}

This material is based in part upon work performed by the Joint Center for Artificial Photosynthesis, a DOE Energy Innovation Hub, supported through the Office of Science of the U. S. Department of Energy under Award Number DE-SC0004993. This material is based upon work supported by the National Science Foundation Graduate Research Fellowship under Grant No. DGE-114747. D.A.T. recognizes a Graduate Research Fellowship from the National Science Foundation for support. 


\section{Author Contributions}

Z.W.U., M.T.T., and M.K. performed the thermodynamic DFT calculations. J.X. performed the transition state calculations. X.L. constructed the microkinetic model. Z.W.U. developed the machine learning regression and refinement methods. D.A.T. and K.C. collected the ARXPS experimental spectra. K.C., C.H., N.S.L., T.F.J., and J.K.N. formulated the problem and approach. All authors contributed to the writing of the manuscript.

\section{Supporting Information Available}

Details of the training scheme for the AMP Neural Network calculator. Details of the density functional theory electronic calculations. Full ARXPS spectra for the characterized $\mathrm{Ni}_{5} \mathrm{Ga}_{3}$ electrodes post-electrolysis, as well as details of the experiment and spectra collection. Selectivity and coverage maps in the microkinetic model.

\section{References}

(1) Chen, J. G.; Menning, C. A.; Zellner, M. B. Surf. Sci. Rep. 2008, 63, $201-254$.

(2) Campbell, C. T. Annu. Rev. Phys. Chem. 1990, 41, 775-837.

(3) Hansen, H.; Shi, C.; Lausche, A.; Peterson, A.; Nørskov, J. Phys. Chem. Chem. Phys. 2016, 18, 9194-9201.

(4) Torelli, D. A.; Francis, S. A.; Crompton, J. C.; Javier, A.; Thompson, J. R.; Brunschwig, B. S.; Soriaga, M. P.; Lewis, N. S. ACS Catal. 2016, 6, 2100-2104.

(5) Liu, X.; Xiao, J.; Peng, H.; Hong, X.; Chan, K.; Nørskov, J. K. Nat. Commun. 2017, 8,15438 . 
(6) Ong, S. P.; Richards, W. D.; Jain, A.; Hautier, G.; Kocher, M.; Cholia, S.; Gunter, D.; Chevrier, V. L.; Persson, K. A.; Ceder, G. Comp. Mater. Sci. 2013, 68, $314-319$.

(7) Zhang, W.-B.; Chen, C.; Zhang, S.-Y. J. Phys. Chem. C 2013, 117, 21274-21280.

(8) Tran, R.; Xu, Z.; Balachandran Radhakrishnan, D. W.; Sun, W.; Persson, K. A.; Ong, S. P. Sci. Data 2016, 3.

(9) Meltzman, H.; Chatain, D.; Avizemer, D.; Besmann, T. M.; Kaplan, W. D. Acta Mater. 2011, 59, $3473-3483$.

(10) Montoya, J. H.; Persson, K. A. npj Comput. Mater. 2017, 3, 14.

(11) Calle-Vallejo, F.; Martnez, J. I.; Garca-Lastra, J. M.; Sautet, P.; Loffreda, D. Angew. Chem., Int. Ed. 2014, 53, 8316-8319.

(12) Ma, X.; Xin, H. Phys. Rev. Lett. 2017, 118, 036101.

(13) Lorenz, S.; Gro, A.; Scheffler, M. Chemical Physics Letters 2004, 395, 210 - 215.

(14) Artrith, N.; Kolpak, A. M. Nano Letters 2014, 14, 2670-2676, PMID: 24742028.

(15) Artrith, N.; Kolpak, A. M. Computational Materials Science 2015, 110, 20 - 28.

(16) Behler, J.; Parrinello, M. Phys. Rev. Lett. 2007, 98, 146401.

(17) Khorshidi, A.; Peterson, A. A. Comp. Phys. Comm. 2016, 20\%, 310-324.

(18) Kolb, B.; Lentz, L. C.; Kolpak, A. M. Sci. Rep. 2017, 7, 1192.

(19) Artrith, N.; Urban, A. Comp. Mater. Sci. 2016, 114, 135 - 150.

(20) Srivastava, N.; Hinton, G.; Krizhevsky, A.; Sutskever, I.; Salakhutdinov, R. J. of Mach. Lear. Res. 2014, 15, 1929-1958. 
(21) Abadi, M.; Agarwal, A.; Barham, P.; Brevdo, E.; Chen, Z.; Citro, C.; Corrado, G. S.; Davis, A.; Dean, J.; Devin, M.; Ghemawat, S.; Goodfellow, I.; Harp, A.; Irving, G.; Isard, M.; Jia, Y.; Jozefowicz, R.; Kaiser, L.; Kudlur, M.; Levenberg, J.; Mané, D.; Monga, R.; Moore, S.; Murray, D.; Olah, C.; Schuster, M.; Shlens, J.; Steiner, B.; Sutskever, I.; Talwar, K.; Tucker, P.; Vanhoucke, V.; Vasudevan, V.; Viégas, F.; Vinyals, O.; Warden, P.; Wattenberg, M.; Wicke, M.; Yu, Y.; Zheng, X. TensorFlow: Large-Scale Machine Learning on Heterogeneous Systems. Proceedings of the 12th USENIX Symposium on Operating Systems Design and Implementation (OSDI). 2016; pp 265-283.

(22) Gal, Y.; Ghahramani, Z. Dropout as a Bayesian approximation: Representing model uncertainty in deep learning. International Conference on Machine Learning (ICML). 2016; pp 1050-1059.

(23) Jain, A.; Ong, S. P.; Chen, W.; Medasani, B.; Qu, X.; Kocher, M.; Brafman, M.; Petretto, G.; Rignanese, G.-M.; Hautier, G. Concurrency and Computation: Practice and Experience 2015, 27, 5037-5059.

(24) Chan, K.; Nørskov, J. K. J. Phys. Chem. Lett. 2016, ๆ, 1686. 


\section{Graphical TOC Entry}

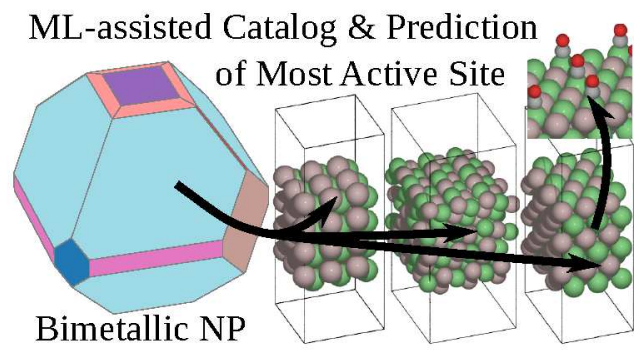

